GLASNIK MATEMATIČKI

Vol. 41(61)(2006), $101-114$

\title{
HOMOGENIZATION OF RANDOM DEGENERATED NONLINEAR MONOTONE OPERATORS
}

\author{
J. Engström, L.-E. Persson, A. Piatnitski and P. Wall \\ Luleå University of Technology, Sweden, Narvik University College, Norway \\ and P. N. Lebedev Physical Institute of RAS, Russia

\begin{abstract}
This paper deals with homogenization of random nonlinear monotone operators in divergence form. We assume that the structure conditions (strict monotonicity and continuity conditions) degenerate and are given in terms of a weight function. Under proper integrability assumptions on the weight function we construct the effective operator and prove the homogenization result.
\end{abstract}

\section{INTRODUCTION}

Mathematical description of microscopically heterogeneous media usually involves rapidly oscillating functions of the form $a=a(x / \varepsilon)$ where $\varepsilon$ is a small positive parameter characterizing the microscopic length scale of the media. The aim of homogenization theory is to provide the macroscopic rigorous description of the studied media. Homogenization is at present a well developed area and there is a vast literature on the topic, see e.g. [1]-[8] and [12]-[18].

The homogenization problems for various random structures are widely discussed in the physical and mathematical literature, see e.g. [13] and its bibliography. The first rigorous results for random elliptic operators in divergence form with stochastically homogeneous coefficients were obtained by Kozlov in [14] and independently by Papanicolaou and Varadhan in [19]. Later on, many other random models were investigated, among them are random porous media (see for instance [12]), convection-diffusion problems (see e.g. [3]), nonlinear models (see e.g. [17]) etc. In [6] Bourgeat et al. developed the stochastic version of the two-scale convergence approach.

2000 Mathematics Subject Classification. 35B27, 35B40.

Key words and phrases. Stochastic homogenization, random operators, degenerated monotone operators. 
We study random nonlinear monotone operators in divergence form, which satisfy weighted structure conditions with weight $\Lambda(x)>0$ being a statistically homogeneous random field. Concerning this weight we assume that $\Lambda \in L_{\text {loc }}^{1}\left(\mathbb{R}^{n}\right)$ and $\Lambda^{-1 /(p-1)} \in L_{\text {loc }}^{1}\left(\mathbb{R}^{n}\right)$ and also that some uniform integrability condition of Muckenhoupt type (see Definition 2.2 below) holds. The corresponding Dirichlet problem takes the form $\left(f \in L^{\infty}(Q), Q \subset \mathbb{R}^{n}\right)$ :

$$
\left\{\begin{array}{l}
-\operatorname{div}\left(A\left(x / \varepsilon, D u_{\varepsilon}\right)\right)=f \quad \text { in } Q, \\
u_{\varepsilon} \in W_{0}^{1, p}(Q, \Lambda(x / \varepsilon)),
\end{array}\right.
$$

where $A(x / \varepsilon, \cdot)$ is a statistically homogeneous field which satisfies the degenerated structure conditions (5.2) and (5.3) below, and $Q$ is a regular domain in $\mathbb{R}^{n}$. In the paper we prove the a.s. convergence

$$
\begin{aligned}
u_{\varepsilon} & \rightarrow u \text { weakly in } W_{0}^{1,1}(Q), \\
A\left(x / \varepsilon, D u_{\varepsilon}\right) & \rightarrow b(D u) \text { weakly in } L^{1}(Q)^{n},
\end{aligned}
$$

and show that the limit function $u$ is the unique solution of the following effective equation

$$
\left\{\begin{array}{l}
-\operatorname{div}(b(D u))=f \quad \text { in } \mathrm{Q}, \\
u \in W_{0}^{1, p}(Q) .
\end{array}\right.
$$

The coefficients $b(\xi)$ here are expressed in terms of solutions of an auxiliary problem involving random variables $a(\xi)=A(0, \xi)$. For details see (4.4) and (4.8).

In periodic case similar homogenization results were obtained in [2] where the framework of weighted Sobolev spaces was used. We believe that one can make use of the singular measure approach developed in $[5,16,20,21]$, to investigate the problems of this type.

Notice that for non-degenerated random operators stronger convergence holds, namely (see e.g. [11] or [17]):

$$
\begin{aligned}
u_{\varepsilon} & \rightarrow u \text { weakly in } W_{0}^{1, p}(Q), \\
A\left(x / \varepsilon, D u_{\varepsilon}\right) & \rightarrow b(D u) \text { weakly in } L^{p}(Q)^{n} .
\end{aligned}
$$

The paper is organized as follows: Section 2 contains the setup and some technical statements. In section 3 we define the class of potential vectorfunctions in a weighted space; then in section 4 we introduce an auxiliary stochastic problem and construct the formal homogenized operator; finally in the last section the homogenization result is proved.

\section{Notation AND PRELIMINARIES}

First we recall the notion of random dynamical system. Let $(\Omega, \mathcal{F}, \mu)$ be a probability space. A family of measurable mappings $T_{x}: \Omega \rightarrow \Omega\left(x \in \mathbb{R}^{n}\right)$ 
is called a $n$-dimensional random dynamical system if it satisfies the following properties:

1. $T_{0}=I$ (i.e. $T_{0}$ is the identity mapping) and

$$
\left.T_{x+y}=T_{x} T_{y} \text { (for every } x, y \in \mathbb{R}^{n}\right) .
$$

2. The map $T_{x}: \Omega \rightarrow \Omega$ preserves the measure $\mu$ i.e. for every $x \in \mathbb{R}^{n}$ and every $\mathcal{U} \in \mathcal{F}$

$$
\mu(\mathcal{U})=\mu\left(T_{x}(\mathcal{U})\right) .
$$

3. For any measurable function $f$ on $\Omega$, the function $f\left(T_{x} \omega\right)$ defined on $\mathbb{R}^{n} \times \Omega$ is measurable $\left(\mathbb{R}^{n} \times \Omega\right.$ is endowed with the product $\sigma$-algebra $\mathcal{B} \times \mathcal{F}$, where $\mathcal{B}$ stands for the Borel $\sigma$-algebra).

Given such a dynamical system we can introduce a wide class of statistically homogeneous random fields. Indeed, let $f: \Omega \rightarrow \mathbb{R}^{n}$ be a random function. Then the function $F(x)=f\left(T_{x} \omega\right)$ is a statistically homogeneous random field. If $\omega \in \Omega$ is fixed, the function $F(x)$ is called a realization of $f$.

We say that $f=f(\omega)$ is invariant if

$$
f(\omega)=f\left(T_{x} \omega\right) \text { a.e. in } \Omega,
$$

for every $x \in \mathbb{R}^{n}$. A dynamical system is ergodic if every invariant function is constant a.s. We assume in the rest of this work that the dynamical system $T_{x}$ is ergodic. The following result will be useful later (for the proof see [13]).

Lemma 2.1. Let $\Omega_{0}$ be a measurable subset of $\Omega$ such that $\mu\left(\Omega_{0}\right)=1$. Then there exists a measurable subset $\Omega_{1} \subset \Omega_{0}$ such that $\mu\left(\Omega_{1}\right)=1$ and for any $\omega \in \Omega_{1}$ we have $T_{x} \omega \in \Omega_{0}$ for a.e. $x \in \mathbb{R}^{n}$.

Now we proceed by introducing weight functions and weighted spaces. Assume that $\lambda: \Omega \rightarrow \mathbb{R}$ is a measurable function such that $\lambda>0$ a.s. and

$$
\lambda \in L^{1}(\Omega), \quad \lambda^{-1 /(p-1)} \in L^{1}(\Omega),
$$

for some $p, 1<p<\infty$. Then by the Fubini theorem almost all realizations satisfy $\Lambda(x)=\lambda\left(T_{x} \omega\right)>0$ a.e. and

$$
\Lambda \in L_{\text {loc }}^{1}\left(\mathbb{R}^{n}\right), \quad \Lambda^{-1 /(p-1)} \in L_{\text {loc }}^{1}\left(\mathbb{R}^{n}\right) .
$$

We denote by $L^{p}(\Omega, \lambda)$ the set of functions $u$ in $L^{1}(\Omega)$ such that $u \lambda^{1 / p} \in$ $L^{p}(\Omega)$, and by $L_{\text {loc }}^{p}\left(\mathbb{R}^{n}, \Lambda\right)$ the set of functions $u \in L_{\text {loc }}^{1}\left(\mathbb{R}^{n}\right)$ such that $u \Lambda^{1 / p} \in L_{\text {loc }}^{p}\left(\mathbb{R}^{n}\right)$. Let $Q$ be a regular bounded domain in $\mathbb{R}^{n}$. Then $W^{1, p}(Q, \Lambda)$ stands for the space of functions $u$ in $W^{1,1}(Q)$ such that $u \in$ $L^{p}(Q, \Lambda)$ and $D u \in L^{p}(Q, \Lambda)^{n}$. Denote by $W_{0}^{1, p}(Q, \Lambda)$ the completion of $C_{0}^{1}(Q)$ in $W^{1, p}(Q, \Lambda)$ with respect to the norm

$$
\left(\int_{Q}\left(|u|^{p}+|D u|^{p}\right) \Lambda d x\right)^{1 / p} .
$$


The conditions (2.2) are rather natural but not sufficient for our purposes. We will impose a stronger version of these conditions, namely the so-called Muckenhoupt condition. For the reader's convenience we formulate it below.

Definition 2.2. Let $K \geq 1$ and let $\Lambda$ be a positive function on $\mathbb{R}^{n}$. Then $\Lambda$ belongs to the class $A_{p}(K)$ if for every cube $Q^{\square} \subset \mathbb{R}^{n}$ with faces parallel to the coordinate planes the following condition is satisfied:

$$
\left(\frac{1}{\left|Q^{\square}\right|} \int_{Q^{\square}} \Lambda d x\right)\left(\frac{1}{\left|Q^{\square}\right|} \int_{Q^{\square}} \Lambda^{-\frac{1}{p-1}} d x\right)^{p-1} \leq K .
$$

Here and in what follows $|B|$ stands for the Lebesgue measure of a Borel set B. We also define $A_{p}=\cup_{K \geq 1} A_{p}(K)$.

Now we define the set of weights used in this paper.

Definition 2.3. The class $\mathcal{N}_{K}^{p}$ is defined as the set of positive functions $\lambda: \Omega \rightarrow \mathbb{R}$ whose realizations belong to $A_{p}(K)$ a.s.

Some properties of the weight functions satisfying the Muckenhoupt condition, are given by the following statement proved in [9]. Throughout this paper we denote by $C$ a generic positive constant which may take on different values in different expressions.

Lemma 2.4. Let $K \geq 1$. Then there exist two positive constants $\delta=$ $\delta(n, p, K)$ and $C=C(n, p, K)$ such that for every cube $Q^{\square} \subset \mathbb{R}^{n}$ with faces parallel to the coordinate planes and every $\Lambda \in A_{p}(K)$

$$
\begin{gathered}
\left(\frac{1}{\left|Q^{\square}\right|} \int_{Q^{\square}} \Lambda^{1+\delta} d y\right)^{\frac{1}{1+\delta}} \leq C \frac{1}{\left|Q^{\square}\right|} \int_{Q^{\square}} \Lambda d y, \\
\left(\frac{1}{\left|Q^{\square}\right|} \int_{Q^{\square}} \Lambda^{-(1+\delta) /(p-1)} d y\right)^{\frac{1}{1+\delta}} \leq C \frac{1}{\left|Q^{\square}\right|} \int_{Q^{\square}} \Lambda^{-1 /(p-1)} d y .
\end{gathered}
$$

We end this section by formulating a version of compensated compactness lemma, adapted to the framework of weighted spaces. For the proof see [2].

Lemma 2.5 (Compensated compactness). Let $\nu \in A_{p}, K \geq 1$, and let $Q$ be an open bounded subset of $\mathbb{R}^{n}$. Given a family of weights $\left\{\Lambda_{\varepsilon}: \Lambda_{\varepsilon} \in\right.$ $\left.A_{p}(K), \varepsilon>0\right\}$, suppose that $\left\{u_{\varepsilon}\right\}$ is a family of functions such that

1. $\int_{Q}\left|D u_{\varepsilon}\right|^{p} \Lambda_{\varepsilon} d y \leq C_{1}<\infty$ for all $\varepsilon>0$,

2. there is $u \in W^{1, p}(Q, \nu)$ such that $u_{\varepsilon} \rightarrow u$ in $L^{1}(Q)$, and $\left(A_{\varepsilon}\right)$ is a family of vector functions in $\mathbb{R}^{n}$ such that

3. $\int_{Q}\left|A_{\varepsilon}\right|^{q} \Lambda_{\varepsilon}^{-1 /(p-1)} d y \leq C_{2}<\infty$ for all $\varepsilon>0$, where $q=p /(p-1)$, 4. there exists $g \in L^{\infty}(Q)$ such that $\operatorname{div}\left(A_{\varepsilon}\right)=g$ in $Q$ for every $\varepsilon>0$, 
5. there is $A \in L^{q}\left(Q, \nu^{-1 /(p-1)}\right)^{n}$ such that $A_{\varepsilon} \rightarrow A$ weakly in $L^{1}(Q)^{n}$. Then

$$
\int_{Q}\left\langle A_{\varepsilon}, D u_{\varepsilon}\right\rangle \phi d y \rightarrow \int_{Q}\langle A, D u\rangle \phi d y
$$

for every $\phi \in C_{0}^{\infty}(Q)$; here and in what follows $\langle\cdot, \cdot\rangle$ stands for the inner product in $\mathbb{R}^{n}$.

\section{Potential functions in a Weighted SPaCe}

Recall that a vector field $f \in L_{\text {loc }}^{p}\left(\mathbb{R}^{n}\right)^{n}$ is said to be potential if there exists a function $u \in W_{\text {loc }}^{1, p}\left(\mathbb{R}^{n}\right)$ such that $f=D u$. A vector field $v$ is said to be solenoidal if $\operatorname{div} v=0$ in the weak sense, i.e.

$$
\int_{\mathbb{R}^{n}}\langle v, D \phi\rangle d x=0 \text { for all } \phi \in C_{0}^{\infty}\left(\mathbb{R}^{n}\right) .
$$

Now we turn to random vector fields. Let us first recall the definition of potential and solenoidal random fields in the non-weighted case. A random function $f \in L^{p}(\Omega)^{n}$ is said to be potential if almost all its realizations are potential. Notice that by the Fubini theorem the realizations of $f$ are a.s. elements of $L_{\text {loc }}^{p}\left(\mathbb{R}^{n}\right)^{n}$. Solenoidal random vector field is defined similarly.

In order to define potential vector fields in a weighted probability space, notice that due to (2.1) and Hölder inequality we have

$$
\int_{\Omega}|f(\omega)| d \mu \leq\left(\int_{\Omega}|f(\omega)|^{p} \lambda(\omega) d \mu\right)^{1 / p}\left(\int_{\Omega}(\lambda(\omega))^{-\frac{1}{p-1}} d \mu\right)^{\frac{p-1}{p}}<\infty,
$$

for any $f \in L^{p}(\Omega, \lambda)^{n}$. Therefore, any element of $L^{p}(\Omega, \lambda)^{n}$ belongs to $L^{1}(\Omega)$ and its realizations belong to $L_{\text {loc }}^{1}\left(\mathbb{R}^{n}\right)$.

It is then natural to say that a vector field $f \in L^{p}(\Omega, \lambda)^{n}$ is potential if almost all its realizations are potential vector fields in $L_{\text {loc }}^{1}\left(\mathbb{R}^{n}\right)^{n}$. We denote this space by $\mathbf{L}_{\text {pot }}^{p}(\Omega, \lambda)^{n}$. We also define

$$
\mathbf{V}_{p o t}^{p}(\Omega, \lambda)=\left\{f \in \mathbf{L}_{\text {pot }}^{p}(\Omega, \lambda): \int_{\Omega} f d \mu=0\right\}
$$

Note that since convergence in $L^{p}(\Omega, \lambda)$ implies convergence for a subsequence of almost all realizations in $L_{\text {loc }}^{1}\left(\mathbb{R}^{n}\right)$, the space $\mathbf{V}_{\text {pot }}^{p}(\Omega, \lambda)$ is closed in $L^{p}(\Omega, \lambda)^{n}$.

Lemma 3.1. Let $f \in \mathbf{L}_{\text {pot }}^{p}(\Omega, \lambda)$, and suppose that $\lambda \in \mathcal{N}_{K}^{p}$. Then there is $\sigma=\sigma(n, p, K)>0$ such that the realizations of $f$ a.s. belong to $L_{\mathrm{loc}}^{1+\sigma}\left(\mathbb{R}^{n}\right)^{n}$.

Proof. If $f \in L^{p}(\Omega, \lambda)^{n}$ then by the Fubini theorem $F(x)=f\left(T_{x} \omega\right) \in$ $L_{\text {loc }}^{p}\left(\mathbb{R}^{n}, \Lambda\right)^{n}$ a.s. Let $Q \subset \mathbb{R}^{n}$, and take $\delta>0$ such that (2.4) holds. We 
choose $\sigma$ so that the relation holds

$$
\frac{1+\sigma}{p-1-\sigma}=\frac{1+\delta}{p-1}
$$

It is easy to check that $\sigma>0$ and $p-1-\sigma>0$. Let $Q^{\square}$ be a cube in $R^{n}$ containing $Q$. The Hölder inequality then gives

$$
\begin{aligned}
\int_{Q}|F|^{1+\sigma} d x & \leq\left(\int_{Q}|F|^{p} \Lambda_{\varepsilon} d x\right)^{\frac{1+\sigma}{p}}\left(\int_{Q} \Lambda_{\varepsilon}^{-\frac{1+\sigma}{p-1-\sigma}} d x\right)^{\frac{p-1-\sigma}{p}} \\
& \leq\left(\int_{Q}|F|^{p} \Lambda_{\varepsilon} d x\right)^{\frac{1+\sigma}{p}}\left(\int_{Q^{\square}} \Lambda_{\varepsilon}^{-\frac{1+\delta}{p-1}} d x\right)^{\frac{p-1}{p+\delta}} .
\end{aligned}
$$

By applying (2.4) in this inequality, we obtain

$\int_{Q}|F|^{1+\sigma} d x \leq\left(\int_{Q}|F|^{p} \Lambda_{\varepsilon} d x\right)^{\frac{1+\sigma}{p}}\left(\int_{Q^{\square}} \Lambda_{\varepsilon}^{1 /(1-p)} d x\right)^{(p-1)(1+\delta) /(p+\delta)}<\infty$ which implies the desired statement.

\section{Auxiliary Results}

In this section we introduce an auxiliary problem and study its properties. The solution of the auxiliary problem is then used when we define the effective operator.

Let $(\Omega, \mathcal{F}, \mu)$ be a probability space, $T_{x}, x \in \mathbb{R}^{n}$, an ergodic dynamical system on $\Omega$, and $Q$ a regular bounded domain in $\mathbb{R}^{n}$. Assume that $\lambda \in \mathcal{N}_{K}^{p}$, and let $a: \Omega \times \mathbb{R}^{n} \rightarrow \mathbb{R}^{n}$ be a measurable function that satisfies the following structure conditions: there are constants $\alpha$ and $\beta$ with $0<\alpha \leq \min \{1, p-1\}$ and $\max \{p, 2\} \leq \beta<\infty$ such that

$$
\begin{gathered}
\left\langle a\left(\omega, \xi_{1}\right)-a\left(\omega, \xi_{2}\right), \xi_{1}-\xi_{2}\right\rangle \geq C \lambda(\omega)\left(1+\left|\xi_{1}\right|+\left|\xi_{2}\right|\right)^{p-\beta}\left|\xi_{1}-\xi_{2}\right|^{\beta} \\
\left|a\left(\omega, \xi_{1}\right)-a\left(\omega, \xi_{2}\right)\right| \leq C \lambda(\omega)\left(1+\left|\xi_{1}\right|+\left|\xi_{2}\right|\right)^{p-1-\alpha}\left|\xi_{1}-\xi_{2}\right|^{\alpha}
\end{gathered}
$$

for every $\xi_{1}, \xi_{2} \in \mathbb{R}^{n}$ a.s. We also assume that

$$
a(\omega, 0)=0 \text { a.s. }
$$

For $\xi \in \mathbb{R}^{n}$ we define the operator $\mathcal{A}^{\xi}$ which maps the space $\mathbf{V}_{\text {pot }}^{p}(\Omega, \lambda)$ into its dual, by

$$
\left\langle\mathcal{A}^{\xi} v, \phi\right\rangle=\int_{\Omega}\langle a(\omega, \xi+v(\omega)), \phi\rangle d \mu \text { for all } \phi \in \mathbf{V}_{p o t}^{p}(\Omega, \lambda) .
$$

Consider the following auxiliary problem: find $v^{\xi} \in \mathbf{V}_{\text {pot }}^{p}(\Omega, \lambda)$ such that

$$
\left\langle\mathcal{A}^{\xi} v^{\xi}, \phi\right\rangle=0 \text { for all } \phi \in \mathbf{V}_{p o t}^{p}(\Omega, \lambda) \text {. }
$$

According to [15, Chapter 2, Theorem 2.1], this problem has a unique solution $v^{\xi}$. Denote by $V^{\xi}$ a realization of $v^{\xi}$, i.e. $V^{\xi}(x)=v^{\xi}\left(T_{x} \omega\right), x \in \mathbb{R}^{n}$. 
By the definition of $\mathbf{V}_{\text {pot }}^{p}(\Omega, \lambda)$ there exists a function $N^{\xi} \in W_{\text {loc }}^{1,1+\sigma}\left(\mathbb{R}^{n}\right)$ such that $V^{\xi}(x)=D N^{\xi}(x)$ a.s. The function $N^{\xi}$ is defined up to an additive constant. We define this additive constant for each $\varepsilon$ by setting

$$
N_{\varepsilon}^{\xi}(x)=N^{\xi}(x)-\frac{1}{|Q|} \int_{Q} N^{\xi}\left(\frac{x}{\varepsilon}\right) d x .
$$

This normalization condition implies the relation

$$
\int_{Q} N_{\varepsilon}^{\xi}\left(\frac{x}{\varepsilon}\right) d x=0
$$

Then using the Poincare inequality we obtain

$$
\left\|\varepsilon N_{\varepsilon}^{\xi}\left(\frac{x}{\varepsilon}\right)\right\|_{L^{1+\sigma}(Q)} \leq C
$$

Thus $\left(\varepsilon N_{\varepsilon}^{\xi}\left(\frac{x}{\varepsilon}\right)\right)$ converges weakly in $L^{1+\sigma}(Q)$ along a subsequence. The limit function must be equal to 0 because $V^{\xi}\left(\frac{x}{\varepsilon}\right) \rightarrow 0$ weakly in $L^{1}(Q)$ by the Birkhoff ergodic theorem. Then the whole sequence $\left(\varepsilon N_{\varepsilon}^{\xi}\left(\frac{x}{\varepsilon}\right)\right)$ converges weakly in $L^{1+\sigma}(Q)$ to 0 . Let us define

$$
w_{\varepsilon}^{\xi}(x)=\langle\xi, x\rangle+\varepsilon N_{\varepsilon}^{\xi}\left(\frac{x}{\varepsilon}\right) .
$$

Since $w_{\varepsilon}^{\xi}(x) \rightarrow\langle\xi, x\rangle$ in $L^{1+\sigma}(Q)$ and $D w_{\varepsilon}^{\xi}(x) \rightarrow \xi$ in $L^{1+\sigma}(Q)^{n}$, we conclude that

$$
w_{\varepsilon}^{\xi}(x) \rightarrow\langle\xi, x\rangle \text { in } L^{1+\sigma}(Q) .
$$

For the sake of brevity for the realizations of $a\left(\omega, \xi+v^{\xi}(\omega)\right)$ we use the notation

$$
F^{\xi}(x)=a\left(T_{x} \omega, \xi+v^{\xi}\left(T_{x} \omega\right)\right), \quad x \in \mathbb{R}^{n} .
$$

From (4.2)-(4.3) we obtain $a\left(\omega, \xi+v^{\xi}(\omega)\right) \in L^{1}(\Omega)^{n}$. Together with the Birkhoff ergodic theorem this gives the convergence

$$
F^{\xi}\left(\frac{x}{\varepsilon}\right) \rightarrow \int_{\Omega} a\left(\omega, \xi+v^{\xi}(\omega)\right) d \mu \quad \text { in } L_{\mathrm{loc}}^{1}\left(\mathbb{R}^{n}\right)^{n} \text { a.s. }
$$

The limit in (4.7) defines the effective operator $b: \mathbb{R}^{n} \rightarrow \mathbb{R}^{n}$ by

$$
b(\xi)=\int_{\Omega} a\left(\omega, \xi+v^{\xi}(\omega)\right) d \mu .
$$

In the same way as in [16] one can prove that the operator $b$ satisfies the structure conditions

$$
\begin{gathered}
\left\langle b\left(\xi_{1}\right)-b\left(\xi_{2}\right), \xi_{1}-\xi_{2}\right\rangle \geq C\left(1+\left|\xi_{1}\right|+\left|\xi_{2}\right|\right)^{p-\beta}\left|\xi_{1}-\xi_{2}\right|^{\beta} \\
\left|b\left(\xi_{1}\right)-b\left(\xi_{2}\right)\right| \leq C\left(1+\left|\xi_{1}\right|+\left|\xi_{2}\right|\right)^{p-1-\frac{\alpha}{\beta-\alpha}}\left|\xi_{1}-\xi_{2}\right|^{\frac{\alpha}{\beta-\alpha}} .
\end{gathered}
$$


The following lemma shows how the equation (4.4) reads in the space of realizations.

Lemma 4.1. Let $w_{\varepsilon}^{\xi}(x)$ be the function defined in (4.5). Then a.s.

$$
\operatorname{div}\left(a\left(T_{x / \varepsilon} \omega, D w_{\varepsilon}^{\xi}(x)\right)\right)=0 \text { on } C_{0}^{1}(Q),
$$

i.e.

$$
\int_{Q}\left\langle a\left(T_{x / \varepsilon} \omega, D w_{\varepsilon}^{\xi}(x)\right), D \phi\right\rangle d x=0 \text { for all } \phi \in C_{0}^{1}(Q) .
$$

Proof. Consider an even function $K(x)$ such that

$$
K(x) \in C_{0}^{\infty}\left(\mathbb{R}^{n}\right), \int_{\mathbb{R}^{n}} K(x) d x=1, K \geq 0 .
$$

Let $K^{\delta}(x)=\delta^{-n} K\left(\delta^{-1} x\right)(\delta>0)$. Then (4.4) gives

$$
\int_{\Omega}\left\langle a\left(\omega, \xi+v^{\xi}(\omega)\right), D_{\omega}\left(K^{\delta} * \phi(\omega)\right)\right\rangle d \mu=0 \text { for all } \phi \in L^{\infty}(\Omega),
$$

where $D_{\omega}$ denotes the stochastic gradient (see e.g. [6]), and

$$
\left(K^{\delta} * \phi\right)(\omega)=\int_{\mathbb{R}^{n}} K^{\delta}(x) \phi\left(T_{x} \omega\right) d x .
$$

This implies

$$
\int_{\Omega}\left\langle a\left(\omega, \xi+v^{\xi}(\omega)\right),\left(D K^{\delta}\right) * \phi(\omega)\right\rangle d \mu=0 \text { for all } \phi \in L^{\infty}(\Omega)
$$

and hence

$$
a\left(\omega, \xi+v^{\xi}(\omega)\right)_{i} * \frac{\partial}{\partial x_{i}} K^{\delta}=0 \text { for a.e. } \omega .
$$

Thus

$$
\left\langle D_{\omega}, a\left(\omega, \xi+v^{\xi}(\omega)\right) * K^{\delta}\right\rangle=0,
$$

which implies

$$
\frac{\partial}{\partial x_{i}}\left(a\left(T_{x} \omega, \xi+v^{\xi}\left(T_{x} \omega\right)\right) * K^{\delta}\right)_{i}=0,
$$

or in weak formulation

$$
\int_{\mathbb{R}^{n}}\left\langle a\left(T_{x} \omega, \xi+v^{\xi}\left(T_{x} \omega\right)\right) * K^{\delta}(x), D \psi(x)\right\rangle d x=0 \text { for all } \psi \in C_{0}^{\infty}\left(\mathbb{R}^{n}\right) .
$$

Hence

$$
\int_{\mathbb{R}^{n}}\left\langle a\left(T_{x} \omega, \xi+v^{\xi}\left(T_{x} \omega\right)\right), K^{\delta}(x) * D \psi(x)\right\rangle d x=0 \text { for all } \psi \in C_{0}^{\infty}\left(\mathbb{R}^{n}\right)
$$

and since $K^{\delta}(x) * D \psi(x) \rightarrow D \psi(x)$ (as $\delta \rightarrow 0$ ) uniformly, we finally obtain

$$
\int_{\mathbb{R}^{n}}\left\langle a\left(T_{x} \omega, \xi+v^{\xi}\left(T_{x} \omega\right)\right), D \psi(x)\right\rangle d x=0 \text { for all } \psi \in C_{0}^{\infty}\left(\mathbb{R}^{n}\right) .
$$

By density argument we are done. 


\section{MAin RESUlt}

We proceed with our main result. Denote $A(x, \xi)=a\left(\left(T_{x} \omega\right), \xi\right)$ and $\Lambda(x)=\lambda\left(T_{x} \omega\right)$. According to Lemma 2.1 the conditions imposed on $a(\omega, \xi)$, imply that a.s.

$$
\begin{gathered}
\left\langle A\left(x, \xi_{1}\right)-A\left(x, \xi_{2}\right), \xi_{1}-\xi_{2}\right\rangle \geq C \Lambda(x)\left(1+\left|\xi_{1}\right|+\left|\xi_{2}\right|\right)^{p-\beta}\left|\xi_{1}-\xi_{2}\right|^{\beta} \\
\left|A\left(x, \xi_{1}\right)-A\left(x, \xi_{2}\right)\right| \leq C \Lambda(x)\left(1+\left|\xi_{1}\right|+\left|\xi_{2}\right|\right)^{p-1-\alpha}\left|\xi_{1}-\xi_{2}\right|^{\alpha} \\
A(x, 0)=0
\end{gathered}
$$

for all $\xi_{1}, \xi_{2} \in \mathbb{R}^{n}$ and a.e. $x \in \mathbb{R}^{n}$. As a direct consequence of (5.1), (5.2), and (5.3) the following inequalities hold (for a.e. $\omega \in \Omega$ )

$$
\begin{gathered}
|A(x, \xi)| \leq C \Lambda(x)\left(1+|\xi|^{p-1}\right), \\
\Lambda(x)|\xi|^{p} \leq C(\Lambda(x)+\langle A(x, \xi), \xi\rangle),
\end{gathered}
$$

for every $\xi \in \mathbb{R}^{n}$ and a.e. $x \in \mathbb{R}^{n}$.

As above, let $Q$ be a regular bounded domain in $\mathbb{R}^{n}$. We can associate to almost each realization $A(x, \xi)$ a family of monotone operators $\mathcal{A}_{\varepsilon}: W_{0}^{1, p}\left(Q, \Lambda_{\varepsilon}\right) \rightarrow W^{1,-q}\left(Q, \Lambda_{\varepsilon}\right)$ defined by

$$
\mathcal{A}_{\varepsilon} u=-\operatorname{div}\left(A\left(\frac{x}{\varepsilon}, D u\right)\right)
$$

or, in the weak form,

$$
\left\langle\mathcal{A}_{\varepsilon} u, v\right\rangle=\int_{Q}\left\langle A\left(\frac{x}{\varepsilon}, D u\right), D v\right\rangle d x \quad \text { for all } v \in W_{0}^{1, p}\left(Q, \Lambda_{\varepsilon}\right) .
$$

Given $f \in L^{\infty}(Q)$, consider the corresponding Dirichlet boundary value problems:

$$
\left\{\begin{array}{l}
-\operatorname{div}\left(A\left(\frac{x}{\varepsilon}, D u_{\varepsilon}\right)\right)=f, \\
u_{\varepsilon} \in W_{0}^{1, p}\left(Q, \Lambda_{\varepsilon}\right) .
\end{array}\right.
$$

According to [15, Chapter 2, Theorem 2.1], if conditions (5.1)-(5.3) are fulfilled, this problem has a unique solution $u_{\varepsilon} \in W_{0}^{1, p}\left(Q, \Lambda_{\varepsilon}\right)$ for each $\varepsilon>0$.

We want to examine the asymptotic behavior of the solutions of (5.6) as $\varepsilon \rightarrow 0$. The following homogenization result holds:

TheOREm 5.1. Let $u_{\varepsilon}$ be solutions of (5.6), and let $b=b(\xi)$ be defined by (4.8). Then a.s.

$$
\begin{aligned}
u_{\varepsilon} & \rightarrow u \text { weakly in } W_{0}^{1,1}(Q), \\
A\left(\frac{x}{\varepsilon}, D u_{\varepsilon}\right) & \rightarrow b(D u) \text { weakly in } L^{1}(Q)^{n}
\end{aligned}
$$


where $u$ is the unique solution of the effective (homogenized) equation

$$
\left\{\begin{array}{l}
-\operatorname{div}(b(D u))=f \\
u \in W_{0}^{1, p}(Q)
\end{array}\right.
$$

Proof. For the reader's convenience we divide the proof in several steps.

SteP 1: Compactness of $\left\{u_{\varepsilon}\right\}$ and $\left\{A\left(\frac{x}{\varepsilon}, D u_{\varepsilon}\right)\right\}$.

From the Birkhoff ergodic theorem it immediately follows that

$$
\int_{Q} \Lambda_{\varepsilon}(x) d x \leq C, \quad \int_{Q}\left(\Lambda_{\varepsilon}(x)\right)^{-1 /(p-1)} d x \leq C .
$$

By (5.5), (5.6) and (5.8) we get

$$
\begin{aligned}
& \int_{Q}\left|D u_{\varepsilon}\right|^{p} \Lambda_{\varepsilon}(x) d x \leq C\left(\int_{Q} \Lambda_{\varepsilon}(x) d x+\int_{Q} f u_{\varepsilon} d x\right) \\
\leq & C\left(\int_{Q} \Lambda_{\varepsilon}(x) d x+\|f\|_{L^{\infty}(Q)} \int_{Q}\left|D u_{\varepsilon}\right| d x\right) \\
\leq & C\left(1+\left(\int_{Q}\left|D u_{\varepsilon}\right|^{p} \Lambda_{\varepsilon}(x) d x\right)^{1 / p}\right) .
\end{aligned}
$$

This yields

$$
\left\|D u_{\varepsilon}\right\|_{L^{p}\left(Q, \Lambda_{\varepsilon}\right)} \leq C .
$$

Also, (5.4) and (5.9) imply

$$
\left\|A\left(\frac{x}{\varepsilon}, D u_{\varepsilon}\right)\right\|_{L^{q}\left(Q, \Lambda_{\varepsilon}^{-1 /(p-1)}\right)^{n}} \leq C .
$$

Take $\delta>0$ such that (2.4) holds, and then choose $\sigma_{1}$ which satisfies the relation

$$
\frac{1+\sigma_{1}}{p-1-\sigma_{1}}=\frac{1+\delta}{p-1}
$$

One can easily check that $\sigma_{1}>0$ and $p-1-\sigma_{1}>0$. Let $Q^{\square}$ be a cube in $\mathbb{R}^{n}$ containing $Q$. The Hölder inequality and (5.9) gives

$$
\begin{aligned}
\int_{Q}\left|D u_{\varepsilon}\right|^{1+\sigma_{1}} d x & \leq\left(\int_{Q}\left|D u_{\varepsilon}\right|^{p} \Lambda_{\varepsilon} d x\right)^{\frac{1+\sigma_{1}}{p}}\left(\int_{Q} \Lambda_{\varepsilon}^{-\frac{1+\sigma_{1}}{p-1-\sigma_{1}}} d x\right)^{\frac{p-1-\sigma_{1}}{p}} \\
& \leq C\left(\int_{Q} \Lambda_{\varepsilon}^{-\frac{1+\delta}{p-1}} d x\right)^{\frac{p-1}{p+\delta}} \leq C\left(\int_{Q^{\square}} \Lambda_{\varepsilon}^{-\frac{1+\delta}{p-1}} d x\right)^{\frac{p-1}{p+\delta}} .
\end{aligned}
$$

By applying (2.4) and (5.8) in this inequality, we obtain

$$
\int_{Q}\left|D u_{\varepsilon}\right|^{1+\sigma_{1}} d x \leq C\left(\int_{Q^{\square}} \Lambda_{\varepsilon}^{1 /(1-p)} d x\right)^{(p-1)(1+\delta) /(p+\delta)} \leq C .
$$


Next, choose $\delta>0$ such that (2.3) holds, and $\sigma_{2}$ such that

$$
1+\delta=\left(1+\sigma_{2}\right) \frac{q-1}{q-1-\sigma_{2}} .
$$

Then $\sigma_{2}>0$ and $q-1-\sigma_{2}>0$. Using (2.3), by the Hölder inequality we get

$$
\int_{Q}\left|A\left(\frac{x}{\varepsilon}, D u_{\varepsilon}\right)\right|^{1+\sigma_{2}} d x \leq C .
$$

Thus if $\sigma=\min \left(\sigma_{1}, \sigma_{2}\right)$, the families $\left\{u_{\varepsilon}\right\}$ and $\left\{A\left(\frac{x}{\varepsilon}, D u_{\varepsilon}\right)\right\}$ are bounded in $W_{0}^{1,1+\sigma}(Q)$ and $L^{1+\sigma}(Q)^{n}$, respectively. This implies the following convergence along a subsequence

$$
\begin{aligned}
u_{\varepsilon} & \rightarrow u_{*} \text { weakly in } W_{0}^{1,1}(Q), \\
A\left(\frac{x}{\varepsilon}, D u_{\varepsilon}\right) & \rightarrow \eta_{*} \text { weakly in } L^{1}(Q)^{n} .
\end{aligned}
$$

STEP 2: Show that $\operatorname{div} \eta_{*}=-f$.

The weak formulation of problem (5.6) reads

$$
\int_{Q}\left\langle A\left(\frac{x}{\varepsilon}, D u_{\varepsilon}\right), D \phi\right\rangle d x=\int_{Q} f \phi d x \quad \text { for all } \phi \in C_{0}^{1}(Q) .
$$

Considering (5.13) and passing to the limit in this relation as $\varepsilon \rightarrow 0$, we get

$$
\int_{Q}\left\langle\eta_{*}, D \phi\right\rangle d x=\int_{Q} f \phi d x \quad \text { for all } \phi \in C_{0}^{1}(Q) .
$$

Density argument and the fact that $\eta_{*} \in L^{q}(Q)^{n}$ (see (5.18) below) then gives

$$
\int_{Q}\left\langle\eta_{*}, D \phi\right\rangle d x=\int_{Q} f \phi d x \quad \text { for all } \phi \in W_{0}^{1, p}(Q) .
$$

Let us now observe that due to $(5.12),(5.13)$ and $(5.14)$ the theorem will be proved if we show that

$$
\begin{aligned}
& u_{*} \in W_{0}^{1, p}(Q), \\
& \eta_{*}=b\left(D u_{*}\right) \text { a.e. on } Q .
\end{aligned}
$$

STEP 3: Proof of (5.15).

It is sufficient to show that $D u_{*} \in L^{p}(Q)^{n}$. For this purpose let $\phi \in$ $C_{0}(Q)$. The Hölder inequality and (5.9) then give

$$
\begin{aligned}
\left\|D u_{\varepsilon} \phi\right\|_{\left(L^{1}(Q)\right)^{n}} & \leq\left(\int_{Q}\left|D u_{\varepsilon}\right|^{p} \Lambda_{\varepsilon}(x) d x\right)^{\frac{1}{p}}\left(\int_{Q}\left(\Lambda_{\varepsilon}(x)\right)^{-\frac{1}{p-1}}|\phi|^{q} d x\right)^{\frac{1}{q}} \\
& \leq C\left(\int_{Q}\left(\Lambda_{\varepsilon}(x)\right)^{-\frac{1}{p-1}}|\phi|^{q} d x\right)^{\frac{1}{q}} .
\end{aligned}
$$


Taking $\liminf _{\varepsilon \rightarrow 0}$ on both sides of the above inequality and using the weak lower semicontinuity of the norm on the left hand side we obtain by the Birkhoff ergodic theorem

$$
\int_{Q}\left|D u_{*}\right||\phi| d x \leq C\|\phi\|_{L^{q}(Q)} \quad \text { for all } \phi \in C_{0}(Q) .
$$

By the density argument we then have

$$
D u_{*} \in L^{p}(Q)^{n} .
$$

By using (5.10) and arguments similar to those employed in the proof of (5.17) one can also show that

$$
\eta_{*} \in L^{q}(Q)^{n}
$$

STEP 4: Proof of (5.16).

We make use of the following test function defined in (4.5)

$$
w_{\varepsilon}^{\xi}(x)=\langle\xi, x\rangle+\varepsilon N_{\varepsilon}^{\xi}\left(\frac{x}{\varepsilon}\right) .
$$

We are going to apply the compensated compactness lemma, see Lemma 2.5. For the reader's convenience let us summarize what we know about the family $\left\{w_{\varepsilon}^{\xi}\right\}$. By the Birkhoff ergodic theorem we have

$$
\begin{aligned}
\int_{Q}\left|D w_{\varepsilon}^{\xi}\right|^{p} \Lambda_{\varepsilon}(x) d x & \leq C, \\
\int_{Q}\left|A\left(\frac{x}{\varepsilon}, D w_{\varepsilon}^{\xi}\right)\right|^{q} \Lambda_{\varepsilon}(x)^{-1 /(p-1)} d x & \leq C .
\end{aligned}
$$

Also, recall that

$$
\begin{aligned}
w_{\varepsilon}^{\xi} & \rightarrow\langle\xi, x\rangle \text { in } L^{1}(Q), \quad(\text { see }(4.6)) \\
A\left(\frac{x}{\varepsilon}, D w_{\varepsilon}^{\xi}\right) & \rightarrow \int_{\Omega} a\left(\omega, \xi+v^{\xi}(\omega)\right) d \mu=b(\xi) \quad \text { weakly in } L^{1}(Q)^{n}, \quad(\text { see }(4.7)) \\
u_{\varepsilon} & \rightarrow u_{*} \text { in } L^{1}(Q), \quad(\text { see }(5.12)) \\
\eta_{\varepsilon} & \rightarrow \eta_{*} \text { weakly in } L^{1}(Q)^{n}, \quad(\text { see }(5.13))
\end{aligned}
$$

where $\eta_{\varepsilon}$ stands for $A\left(\frac{x}{\varepsilon}, u_{\varepsilon}\right)$. Finally by Lemma 4.1 and due to the equation (5.6) we have

$$
\begin{aligned}
\operatorname{div}\left(A\left(\frac{x}{\varepsilon}, D w_{\varepsilon}^{\xi}(x)\right)\right) & =0 & & \text { on } C_{0}^{1}(Q) \text { for all } \varepsilon>0 \\
\operatorname{div}\left(\eta_{\varepsilon}\right) & =f & & \text { on } C_{0}^{1}(Q) \text { for all } \varepsilon>0 .
\end{aligned}
$$

By the monotonicity of $A$ we have for each $\xi \in \mathbb{R}^{n}$

$$
\int_{Q}\left\langle A\left(\frac{x}{\varepsilon}, D u_{\varepsilon}(x)\right)-A\left(\frac{x}{\varepsilon}, D w_{\varepsilon}^{\xi}(x)\right), D u_{\varepsilon}-D w_{\varepsilon}^{\xi}(x)\right\rangle \phi(x) d x \geq 0
$$


for every $\phi \in C_{0}^{\infty}(Q), \phi \geq 0$. Lemma 2.5 applies on the left hand side here and, passing to the limit, we obtain

$$
\int_{Q}\left\langle\eta_{*}(x)-b(\xi), D u_{*}(x)-\xi\right\rangle \phi(x) d x \geq 0,
$$

for every $\phi \in C_{0}^{\infty}(Q), \phi \geq 0$. Hence, for every $\xi \in \mathbb{R}^{n}$ we have

$$
\left(\eta_{*}(x)-b(\xi), D u_{*}(x)-\xi\right) \geq 0 \text { for a.e. } x \in Q .
$$

By the continuity of $b$, the crucial relation (5.16) is now straightforward.

It remains to notice that due to the uniqueness of a solution of the limit problem (5.7), the whole family $\left\{u_{\varepsilon}\right\}$ converges.

\section{REFERENCES}

[1] G. Allaire, A. Braides, G. Buttazzo, A. Defranceschi and L. Gibiansky, School on Homogenization. Lecture Notes of the Courses held at ICTP, Trieste, 4-7 September 1993. Preprint SISSA, Trieste, 1993.

[2] R. De Arcangelis and F. Serra Cassano, On the homogenization of degenerate elliptic equations in divergence form, J. Math. Pures Appl. (9) 71 (1992), 119-138.

[3] M. Avellaneda and A. Majda, An integral representation and bounds on the effective diffusivity in passive advection by laminar and turbulent flows, Comm. Math. Phys. 138 (1991), 339-391.

[4] A. Bensoussan, J.-L. Lions and G. Papanicolaou. Asymptotic Analysis for Periodic Structures, North Holland, Amsterdam, 1978.

[5] G. Bouchitté and I. Fragalà, Homogenization of thin structures by two-scale method with respect to measures, SIAM J. Math. Anal. 32 (2001), 1198-1226.

[6] A. Bourgeat, A. Mikelić and S. Wright, Stochastic two-scale convergence in the mean and applications, J. Reine Angew. Math. 456 (1994), 19-51.

[7] A. Braides and A. Defranceschi, Homogenization of Multiple Integrals, Oxford University Press, New York, 1998.

[8] D. Cioranescu and P. Donato, An Introduction to Homogenization, Oxford University Press, Oxford, 1999.

[9] R. R. Coifman and C. Fefferman, Weighted norm inequalities for maximal function and singular integrals, Studia Math. 51 (1974), 241-250.

[10] N. Dunford and J. T. Schwartz, Linear Operators, Wiley, New York, 1957.

[11] J. Engström, Some Contribution to Homogenization Theory, Licentiate thesis 2002:34, Luleå University of Technology, Luleå, 2002.

[12] U. Hornung, Homogenization and Porous Media, Springer-Verlag, New York, 1997.

[13] V. V. Jikov, S. M. Kozlov and O. A. Oleinik, Homogenization of Differential Operators and Integral Functionals, Springer-Verlag, Berlin-Heidelberg-New York, 1994.

[14] S. Kozlov, Averaging of random operators, Math. USSR-Sb 37 (1980), 167-180.

[15] J.-L. Lions, Quelques méthodes de résolution des problèmes aux limites non linéaires, Dunod Gauthier-Villars, Paris, 1969.

[16] D. Lukkassen and P. Wall, Two-scale convergence with respect to measures and homogenization of monotone operators, J. Funct. Spaces Appl. 3 (2005), 125-161.

[17] A. Pankov, G-convergence and Homogenization of Nonlinear Partial Differential Operators, Mathematics and Its Applications 422, Kluwer Academic Publishers, London, 1997.

[18] L.-E. Persson, L. Persson. N. Svanstedt and J. Wyller. The Homogenization Method: An Introduction, Studentlitteratur, Lund, 1993. 
[19] S. R. Varadhan, Boundary value problems with rapidly ocsillating random coefficients, Colloq. Math. Soc. János Bolyai 27 (1981), 835-873.

[20] V. V. Zhikov, On a homogenization technique for variational problems, Funct. Anal. Appl. 33 (1999), 11-24.

[21] V. V. Zhikov, On an extension and an application of two-scale convergence method, Math. Sb. 191 (2000), 31-72.

J. Engström

Department of Mathematics

Luleå University of Technology

SE-971 87 Luleå

Sweden

\section{L.-E. Persson}

Department of Mathematics

Luleå University of Technology

SE-971 87 Luleå

Sweden

A. Piatnitski

Narvik University College

N-8505 Narvik

Norway

P. Wall

Department of Mathematics

Luleå University of Technology

SE-971 87 Luleå

Sweden

Received: 13.6.2005.

Revised: 3.9.2005.
P. N. Lebedev Physical Institute of RAS

\& Leninski pr., 53, Moscow 119991

Russia 